\title{
Enhanced Asymmetrically Clipped Optical OFDM for Next Generation PONs
}

\author{
Mohammed M. A. Mohammed, and Ziad A. El-Sahn \\ Photonics Group, Electrical Engineering Department, Alexandria University, Alexandria 21544, Egypt \\ alsarariomhammed@gmail.com, ziad.elsahn@ieee.org
}

\begin{abstract}
We successfully demonstrate a novel enhanced asymmetrically clipped optical OFDM (eACO-OFDM) solution over a PON link. The proposed technique doubles the spectral efficiency of conventional ACO-OFDM at the expense of a slight increase in complexity. OCIS codes: (060.2330) Fiber optics communications; (060.4080) Modulation.
\end{abstract}

\section{Introduction}

Nowadays, emerging Internet protocol TV (IPTV) and real-time online gaming applications are creating an increasing need for consumer high data rates. Fiber-to-the-home based on a passive optical network (PON) infrastructure is recognized as a future-proof solution that alleviates the increasing bandwidth demand in the access while offering ultra-fast connections to end users [1]. Recently, different multiple access techniques have been proposed for next generation PONs (NG-PONs), including wavelength-division multiplexing (WDM), orthogonal frequency-division multiple-access (OFDMA), and others. Among the different solutions, OFDMA is a good candidate for next generation PONs aiming at more than $1 \mathrm{~Gb} / \mathrm{s}$ per user, because of its high spectral efficiency, resilience to chromatic dispersion (CD), and simple equalization techniques $[1,2]$.

In PONs, it is favorable to use intensity modulation/direct detection (IM/DD) transceivers because of their simplicity and low-cost. In IM/DD systems, the transmitted electrical signal directly modulates the intensity of an optical carrier. Therefore, only real and non-negative signals can be used. To generate real signals at the transmitter in IM/DD OFDM systems, it is possible to use an inverse fast Fourier transform (IFFT) which input is constrained to have Hermitian symmetry at the expense of half of the spectral efficiency [3]. DC biased optical OFDM (DCOOFDM) [3], and asymmetrically clipped optical OFDM (ACO-OFDM) [4] are more commonly used to generate nonnegative real OFDM signals. A DC bias is used to make the signal positive in DCO-OFDM, which reduces the power efficiency. ACO-OFDM is used as an alternative power efficient technique that only modulates the odd subcarriers while having zeros on even subcarriers. This creates an asymmetric bipolar signal in time domain, which is clipped later at zero level to obtain a non-negative signal at the expense of a further reduction in spectral efficiency.

In this paper, we propose an enhanced ACO-OFDM (eACO-OFDM) system that avoids the use of Hermitian symmetry, therefore doubling the spectral efficiency of conventional ACO-OFDM system.

\section{Proposed Enhanced ACO-OFDM (eACO-OFDM) for Next Generation PONs}

In conventional ACO-OFDM system, due to Hermitian symmetry, half of the bandwidth is used to carry the conjugate of the complex data symbols, and half of the remaining part is used to carry zeros [3]. Therefore, only one fourth of the available bandwidth, i.e. subcarriers, is used to transmit data while the remaining bandwidth is used to carry

(a)

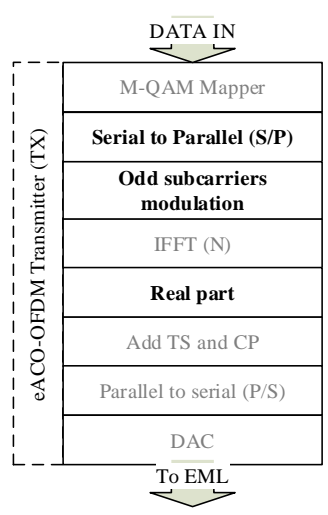

(b)

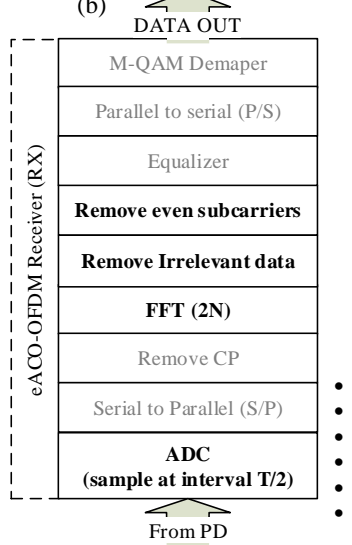

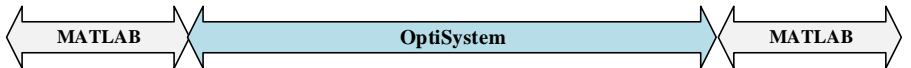

(c)

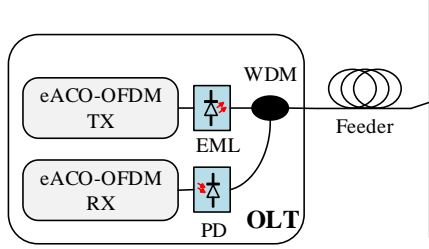

ADC : Analog to digital converter CP : cyclic prefix DAC : Digital to analog converter DDF : Distribution droop fiber EML : Electroabsorption $\bmod$ FFT : Fast Fourier transform

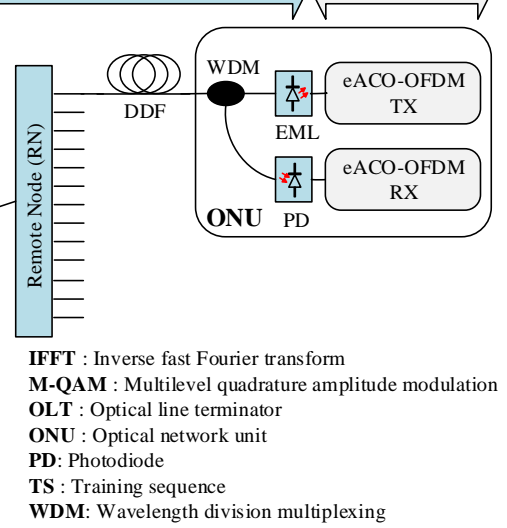

Fig. 1. The proposed eACO-OFDM: (a) transmitter structure, (b) receiver structure, and (c) PON architecture with eACO-OFDM in uplink/downlink directions. 
redundant data or zeros. The eACO-OFDM avoids the use of Hermitian symmetry, therefore allowing data to be carried over half of the available bandwidth compared to only one fourth for conventional ACO-OFDM.

In the proposed eACO-OFDM, after M-QAM data mapping and serial to parallel (S/P) conversion, the signal is passed to the IFFT block with length $\mathrm{N}$ (total number of subcarriers) to transfer the data from frequency to time domain as shown in Fig. 1 (a). The time domain signal is simply given by:

$$
x_{n}=\frac{1}{N} \sum_{k=0}^{N-1} X_{k} e^{\frac{j 2 \pi n k}{N}},
$$

where $X_{k}$ is the $\mathrm{k}^{\text {th }}$ subcarrier of the signal. The IFFT complex output is then split into inphase (real) and quadrature (imaginary) parts, where only the inphase part is transmitted. It has been proved in [5], that the data symbols can be recovered by only sending the inphase part. Assuming that each symbol in the output of the M-QAM mapper is represented by $X_{k}=a_{k}+b_{k}$, where $a_{k}$ and $b_{k}$, are the inphase and quadrature components, respectively, then the inphase part of $x_{n}$ can be expressed as follows

$$
\operatorname{Re}\left\{x_{n}\right\}=\frac{2}{\mathrm{~N}} \sum_{k=0}^{N-1}\left(a_{k} \cos \frac{2 \pi \mathrm{nk}}{N}-b_{k} \sin \frac{2 \pi \mathrm{nk}}{N}\right) .
$$

After appending the CP and a TS, the signal is passed to a DAC before modulating the intensity of the optical carrier.

At the receivesr side in Fig. 1 (b), the signal is converted back to digital for further processing. In order to recover the transmitted data symbols, it is necessary to sample the received signal at intervals $\mathrm{T} / 2$, where $\mathrm{T}$ is the sample interval between the samples of $x_{n}$ as in [5]. Then, after CP removal, the signal is passed to a FFT block with length 2N samples (doubled FFT size compared to ACO-OFDM), which slightly increases the receiver complexity. Assuming no channel distortion, the signal at the FFT output can be given by [5],

$$
Y_{k}=\left\{\begin{array}{lr}
2 a_{0}, & n=0 \\
a_{n}+b_{n}, & 0<n \leq N-1 \\
\text { irrelevant, } & n>N-1
\end{array} .\right.
$$

The original data $a_{n}$ and $b_{n}$ are therefore recovered except for $n=0$, therefore the first subcarrier is modulated with zero. The data is then equalized and recovered after the M-QAM de-mapper.

In the PON configuration in Fig. 1(d); the eACO-OFDM transmitter generates an OFDM signal that directly modulates an electro-absorption modulated laser (EML). The signal is then sent over the DDF, RN and through the feeder to the OLT for uplink or over the feeder, RN, and through DDF to the ONU for downlink. At OLT/ONU, after photodetection, the signal is fed into the eACO-OFDM receiver similar to that in Fig. 1(b).

\section{Performance of the Proposed System}

In this part, the proposed system is tested over both uplink and downlink directions, but focusing more on the uplink direction. The eACO-OFDM signal with 512 FFT length is generated offline using Matlab. The signal is then passed to Optisystem to directly modulate a $1270 \mathrm{~nm}$ uplink EML and a $1577 \mathrm{~nm}$ downlink EML (compatible with XG-PON) that outputs $5 \mathrm{dBm}$ of power. The signal is then sent over the optical distribution network (ODN) in Fig. 1. At the receiver side, after photodetection, the signal is sent back to Matlab for decoding and BER calculation. The BER performance of the eACO-OFDM versus ACO-OFDM at 1.25 Gbaud using 4-QAM and 16-QAM modulation formats over a downlink and uplink PON links are shown in Fig. 2 (a). Both directions almost perform the same for 4-QAM and 16-QAM. However, eACO-OFDM outperforms ACO-OFDM in terms of bandwidth efficiency as explained earlier yielding effective bit rates of $1.25 \mathrm{~Gb} / \mathrm{s}$ for 4-QAM, and $2.5 \mathrm{~Gb} / \mathrm{s}$ for 16-QAM compared to $0.625 \mathrm{~Gb} / \mathrm{s}$ and $1.25 \mathrm{~Gb} / \mathrm{s}$, respectively for ACO-OFDM. The sensitivity curves at the forward error correction (FEC) threshold (BER $=10^{-3}$ ) over a $20 \mathrm{~km}$ feeder as a function of the transmission effective bit rate for both eACO-OFDM and ACO-OFDM using 4-QAM and 16-QAM constellations are depicted in Fig. 2 (b). It can be seen that with the enhanced system, we could achieve the same bit rates as in conventional systems with an improved receiver sensitivity. Using 4-QAM modulation format with $-22 \mathrm{dBm}$ receiver sensitivity at FEC limit the maximum bit rates that could be achieved after $20 \mathrm{~km}$ are $2.5 \mathrm{~Gb} / \mathrm{s}$ with eACO-OFDM and $1.25 \mathrm{~Gb} / \mathrm{s}$ with ACO-OFDM. This leads to a gain of $1.25 \mathrm{~Gb} / \mathrm{s}$ when using the enhanced technique, which means that the obtained effective rate is doubled. On the other hand, $4 \mathrm{~Gb} / \mathrm{s} \mathrm{bit} \mathrm{rate}$ using 4-QAM/eACO-OFDM requires only $-21.5 \mathrm{dBm}$, while 4-QAM/ACO-OFDM requires -19.2 dBm receiver sensitivity, which means a gain of $-2 \mathrm{~dB}$ in receiver sensitivity as shown in Fig. 2 (b). In addition, appropriate modulation order and bitrate could be selected depending on the required receiver sensitivity at FEC limit and required effective bit rate. For example, at $-22 \mathrm{dBm}$ of receiver sensitivity and for a $4 \mathrm{~Gb} / \mathrm{s}$ effective bit rate, 4-QAM would be 
(a)

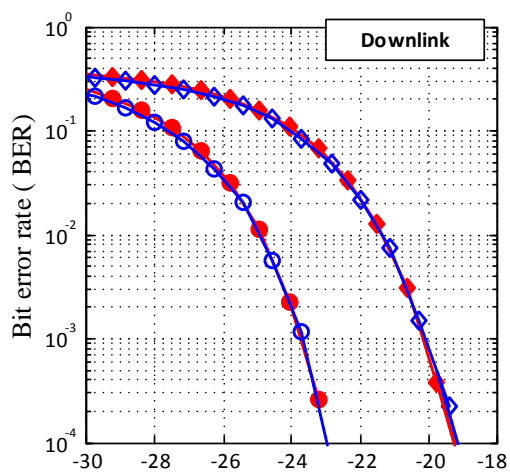

Received optical power $(\mathrm{dBm})$

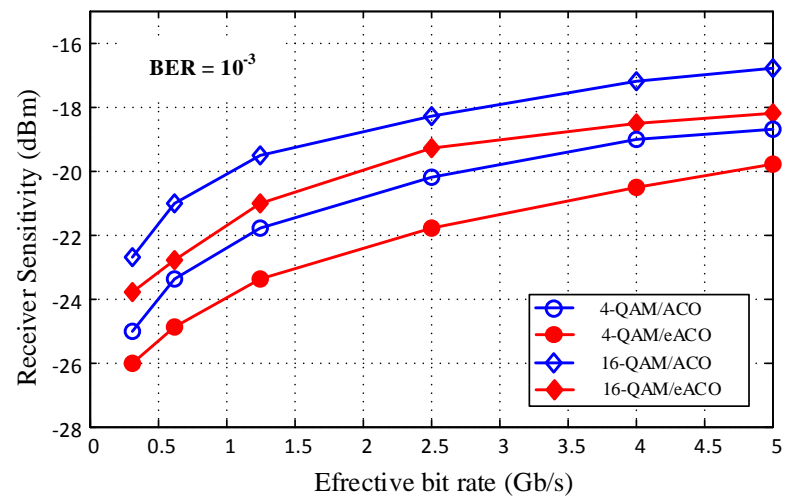

(b)

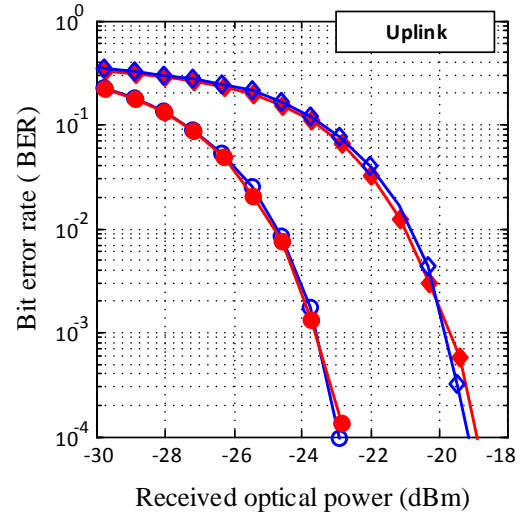

- 4-QAM/ACO $(0.625 \mathrm{~Gb} / \mathrm{s})$

- 4-QAM/eACO $(1.25 \mathrm{~Gb} / \mathrm{s}$ - 16-QAM/ACO $(1.25 \mathrm{~Gb} / \mathrm{s}$ $\sim$ 16-QAM/eACO $(2.5 \mathrm{~Gb} / \mathrm{s})$

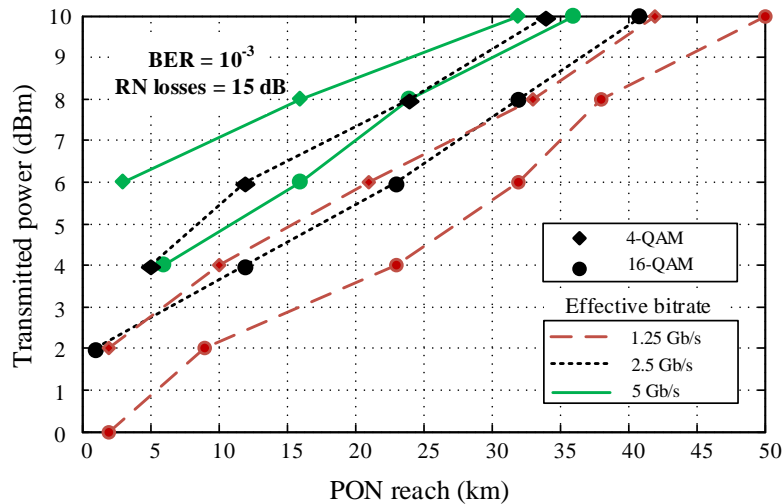

(c)

Fig. 2. (a) Downlink and uplink BER performance over a $20 \mathrm{~km}$ PON for eACO-OFDM and ACO-OFDM using 4-QAM and 16-QAM, (b) eACOOFDM at FEC limit and 32 users with different effective bit rates over uplink direction, (c) Receiver sensitivity plots at FEC limit for different effective bit rates of ACO-OFDM and eACO-OFDM using 4-QAM and 16-QAM over $20 \mathrm{~km}$ PON (uplink).

preferred over the 16-QAM even for eACO-OFDM or ACO-OFDM. Fig. 2(c) shows bit rate contour plots versus the ONU launch power and PON reach at the forward error correction (FEC) limit $\left(\mathrm{BER}=10^{-3}\right)$ for $15 \mathrm{~dB}$ RN losses (corresponding to a 1:32 split ratio) for the proposed eACO-OFDM system. Appropriate modulation order and effective bitrate could be selected depending on the available launch power and the required PON reach. For example, at $6 \mathrm{dBm}$ of launch power and for a PON reach of more than $30 \mathrm{~km}, 4 \mathrm{QAM}$ at $1.25 \mathrm{~Gb} / \mathrm{s}$ would be preferred over the $1.25 \mathrm{~Gb} / \mathrm{s} 16 \mathrm{QAM}$. Also for a $20 \mathrm{~km}$ PON reach, 4-QAM and 16-QAM can achieve $5 \mathrm{~Gb} / \mathrm{s}$ with a $1.5 \mathrm{~dB}$ difference in launch power.

\section{Conclusion}

The enhanced eACO-OFDM is proposed and analyzed over a PON fiber link. The proposed solution doubles the spectral efficiency compared to conventional ACO-OFDM. The simulation results show that the eACO-OFDM performs almost similarly to ACO-OFDM, if they are compared using the same modulation formats. However, the enhanced system outperforms the conventional system if they are fairly compared for the same effective bit rate. Therefore, this gives the proposed system an advantage over the conventional ACO-OFDM IM/DD system.

\section{References}

[1] Neda Cvijetic, “OFDM for next-generation optical access networks,” J. Lightwave Technology, vol. 30, no. 4, February, 2012.

[2] M. M. Mohammed, A. E. El-Fiqi, and Z. A. El-Sahn, "Novel unipolar sign encoded OFDM for next generation PONs," in Proc. Conference on Lasers and Electro-Optics (CLEO), Paper JTh2A.52, San Jose, California, USA, May 2015.

[3] J. Armstrong, and B. J. C. Schmidt, "Comparison of asymmetrically clipped optical OFDM and DC-Biased optical OFDM in AWGN," IEEE Communications Letters, vol. 12, no. 5, pp. 343-345, May 2008.

[4] J. Armstrong, and A. Lowery, "Power efficient optical OFDM," Electronics Letters, vol. 42, no. 6, pp. 370-372, Mar. 16, 2006.

[5] S. B. Weinstein and P. M. Ebert, "Data Transmission for Frequency-Division Multiplexing Using the Discrete Fourier Transform," IEEE Trans. Commun. Tech., vol. 19, no. 5, Oct. 1971, pp. 628-34. 